\title{
Microwave Assisted Synthesis and Biological Evaluation of 1, 2, 4-Triazolo[1, 5-a]pyrimidines
}

\author{
PARTHIV KANTILAL CHAUDHARI \\ Government Science College, Chemistry Department, \\ Vankal-394430 Ta:- Mangrol, Di:- Surat, India \\ parthivkantilal@gmail.com
}

Received 13 October 2016 / Accepted 7 November 2016

\begin{abstract}
A microwave assisted synthesis of three new series of of 1, 2, 4-triazolo[1, 5- $a$ ]pyrimidines (PK-101 to PK-110) has been synthesized by the mixture of 5-(methylthio)-2H-1,2,4-triazol-3-amine (0.01 mol), 4,4,4-trifluoro-1-(4-methoxyphenyl)butane-1,3-dione $(0.01 \mathrm{~mol})$ and an appropriate aromatic aldehyde $(0.01 \mathrm{~mol})$ in ethanol $(5 \mathrm{~mL})$ was irradiated under microwave conditions at $120{ }^{\circ} \mathrm{C}$ for 10-15 min. The structures of all the newly synthesized compounds are elucidated by FT-IR, mass spectra, ${ }^{1} \mathrm{H}$ NMR and elemental analysis. The newly synthesized compounds are subjected to various biological activities viz., antimicrobial, antimycobacterial, anticancer and antiviral.
\end{abstract}

Keywords: 1,2,4-Triazolo[1,5-a]pyrimidines, Antimicrobial activity, Anticancer, Antiviral, Antituberculosis activity, Antimycobacterial activity

\section{Introduction}

The condensation of a ring of 1, 2, 4-triazole and another one of pyrimidine gives rise to the formation of bicyclic heterocycles known as 1, 2, 4-triazolopyrimidines. Four different possibilities exist for the relative orientation of both rings, so four different isomeric families of compounds are defined: 1,2,4-triazolo[1,5-a]pyrimidine (1), 1,2,4-triazolo[1,5-c] pyrimidine (2), 1,2,4-triazolo[4,3-a]pyrimidine (3) and 1,2,4-triazolo[4,3-c]pyrimidine (4).

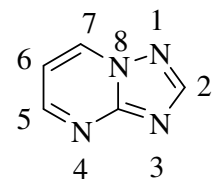

(1) 1,2,4-triazolo[1,5-a]pyrimidine<smiles>c1cnc2nncn2c1</smiles>

(3) 1,2,4-triazolo[4,3-a]pyrimidine<smiles>Cc1nncn2cncc12</smiles>

(2) 1,2,4-triazolo[1,5-c]pyrimidine

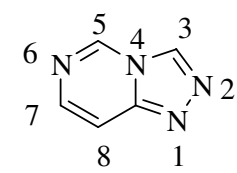

(4) 1,2,4-triazolo[4,3-c]pyrimidine 
Among these isomeric families of compounds, 1, 2, 4-triazolo[1, 5-a]pyrimidine derivatives are thermodynamically more stable and thus, the most studied ones ${ }^{1}$, a few of them being commercially available. Revisions surveying the synthesis, reactivity, spectroscopic characterization and crystallographic studies of 1,2,4-triazolo[1,5-c] pyrimidines $^{2}$, 1,2,4-triazolo[4,3-a]pyrimidines ${ }^{3}$ and 1,2,4-triazolo[4,3-c]pyrimidines ${ }^{4}$ have also been published.

The 1,2,4-triazolo[1,5-a]pyrimidines have aroused increasing attention from the chemical and biological view points, due to their diverse pharmacological activities, such as antitumor potency ${ }^{5,6}$, inhibition of KDR kinase ${ }^{7}$, antifungal effect ${ }^{8}$ and macrophage activation ${ }^{9}$. They have proved to be promising anticancer agents with dual mechanisms of tubulin polymerization promotion as well as cycling dependent kinases 2 inhibition ${ }^{10}$.

By far the most triazolo[1,5-a]pyrimidine synthesis are condensations of dinucleophilic 5amino-1,2,4-triazoles with 1,3-bifunctional synthons as shown in the formation of triazolo[1,5-a] pyrimidine (5) (Scheme 1) ${ }^{11-13}$. New synthetic conditions recently described involve melting under microwave irradiation, a reaction that is environmental friendly and gives higher yields than conventional heating in solvent ${ }^{14}$. Furthermore, certain lithium 1,3-diketonates have proven to be better synthons than the corresponding diketones ${ }^{15}$.<smiles>CCOC(=O)CC(C)=O</smiles>

Scheme 1

Previous mechanistic conclusions have been confirmed by isolating stable intermediate 5-amino-1, 2, 4-triazole derivatives such as enamine (6) (Scheme 2) on reacting 5-amino-1, 2, 4-triazoles with 3-ketovinyl ethers ${ }^{16}$, 3-ketoenamines ${ }^{17}$, 3-ketoaldehydes ${ }^{18}$, enamine-2carboxylic esters $^{19}$ or ethoxymethylene malonates ${ }^{20}$.

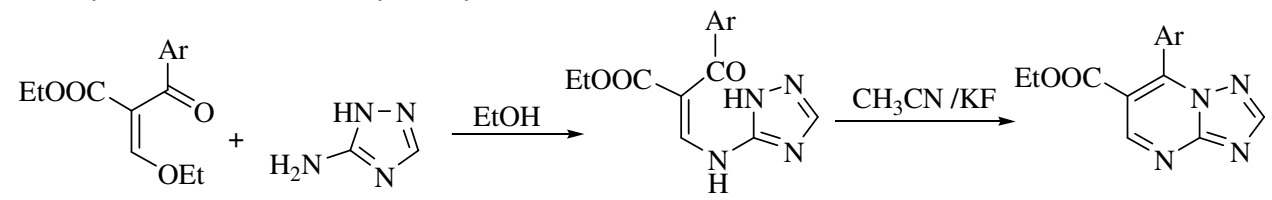

\section{Current work}

Scheme 2 (6)

Microwave assisted organic synthesis has become an important tool to medicinal chemists for rapid organic synthesis. A huge number of research papers have appeared over the last decades on the application of microwave technology in organic synthesis. Some of the major advantages of microwave assisted organic synthesis include spectacular decrease in reaction time, improved conversions, clean product formation and wide scope for the development of new reaction conditions.

The biological importance of 1, 2, 4-triazolo [1, 5-a]pyrimidines is well reported. Over the years, various substituted derivatives of these heterocycles have shown utility against a range of biological targets. For example, they have demonstrated activity against malaria and bronchospasm and shown activity as coronary vasodilators, antihypertensive agents, leishmanicides, antibiotics, adenosine $\mathrm{A}_{2}$ an antagonists, immunosuppressants, antitumor agents, fungicides, xanthine oxidase inhibitors and phosphodiesterase inhibitors. 
The microwave assisted synthesis of three new series of 1, 2, 4-triazolo[1,5a]pyrimidines (PK-101 to PK-110) has been undertaken. The structures of all the newly synthesized compounds are elucidated by FT-IR, mass spectra, ${ }^{1} \mathrm{H}$ NMR and elemental analysis. The newly synthesized compounds are subjected to various biological activities viz., antimicrobial, antimycobacterial, anticancer and antiviral.

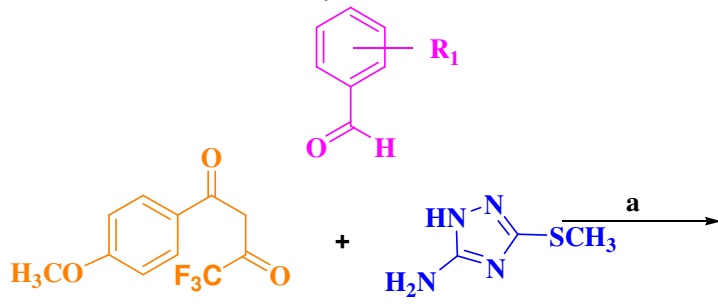

Reagents and conditions: (a) EtOH, MW, $120^{\circ} \mathrm{C}, 10-15 \mathrm{~min}$<smiles>[R]c1cccc(C2C(C(=O)c3ccc(OC)cc3)=C(C(F)(F)F)Nc3nc(SC)nn32)c1</smiles>

PK-101 T0 110

\section{Scheme 3}

Table 1. Physical and analytical data

\begin{tabular}{cccccccc}
\hline Code & \multirow{2}{*}{$\mathrm{R}_{1}$} & M.F. & M.W. & M.P. ${ }^{\circ} \mathrm{C}$ & $\begin{array}{c}\text { Yield } \\
\%\end{array}$ & \multirow{2}{*}{$\mathrm{R}_{\mathrm{f} 1}$} & $\mathrm{R}_{\mathrm{f} 2}$ \\
\hline PK-101 & $4-\mathrm{OCH}_{3}$ & $\mathrm{C}_{22} \mathrm{H}_{19} \mathrm{~F}_{3} \mathrm{~N}_{4} \mathrm{O}_{3} \mathrm{~S}$ & 476 & $221-227$ & 78 & 0.55 & 0.73 \\
PK-102 & $4-\mathrm{F}$ & $\mathrm{C}_{21} \mathrm{H}_{16} \mathrm{~F}_{4} \mathrm{~N}_{4} \mathrm{O}_{2} \mathrm{~S}$ & 464 & $228-230$ & 72 & 0.52 & 0.70 \\
PK-103 & $4-\mathrm{CH}_{3}$ & $\mathrm{C}_{22} \mathrm{H}_{19} \mathrm{~F}_{3} \mathrm{~N}_{4} \mathrm{O}_{2} \mathrm{~S}$ & 460 & $198-200$ & 53 & 0.45 & 0.66 \\
PK-104 & $4-\mathrm{NO}_{2}$ & $\mathrm{C}_{21} \mathrm{H}_{16} \mathrm{~F}_{3} \mathrm{~N}_{5} \mathrm{O}_{4} \mathrm{~S}$ & 491 & $235-237$ & 63 & 0.56 & 0.65 \\
PK-105 & $4-\mathrm{Cl}$ & $\mathrm{C}_{21} \mathrm{H}_{16} \mathrm{ClF}_{3} \mathrm{~N}_{4} \mathrm{O}_{2} \mathrm{~S}$ & 480 & $240-242$ & 75 & 0.55 & 0.75 \\
PK-106 & $3-\mathrm{Cl}$ & $\mathrm{C}_{21} \mathrm{H}_{16} \mathrm{ClF}_{3} \mathrm{~N}_{4} \mathrm{O}_{2} \mathrm{~S}$ & 480 & $188-190$ & 68 & 0.44 & 0.71 \\
PK-107 & $3-\mathrm{NO}_{2}$ & $\mathrm{C}_{21} \mathrm{H}_{16} \mathrm{~F}_{3} \mathrm{~N}_{5} \mathrm{O}_{4} \mathrm{~S}$ & 491 & $256-258$ & 65 & 0.52 & 0.72 \\
PK-108 & $2-\mathrm{Cl}$ & $\mathrm{C}_{21} \mathrm{H}_{16} \mathrm{ClF}_{3} \mathrm{~N}_{4} \mathrm{O}_{2} \mathrm{~S}$ & 480 & $260-262$ & 72 & 0.48 & 0.65 \\
PK-109 & $2-\mathrm{NO}_{2}$ & $\mathrm{C}_{21} \mathrm{H}_{16} \mathrm{~F}_{3} \mathrm{~N}_{5} \mathrm{O}_{4} \mathrm{~S}$ & 491 & $255-257$ & 70 & 0.44 & 0.60 \\
PK-110 & $2-\mathrm{OCH}_{3}$ & $\mathrm{C}_{22} \mathrm{H}_{19} \mathrm{~F}_{3} \mathrm{~N}_{4} \mathrm{O}_{3} \mathrm{~S}$ & 476 & $221-223$ & 60 & 0.45 & 0.76 \\
\hline
\end{tabular}

TLC Solvent system $R_{f 1}$ : Hexane: Ethyl acetate - 5:5; TLC Solvent system $R_{f 2}$ : Chloroform: Methanol - 9:1

\section{Experimental}

Melting points were determined in open capillary tubes and are uncorrected (Table 1). Formation of the compounds was routinely checked by TLC on silica gel-G plates of 0.5 $\mathrm{mm}$ thickness and spots were located by iodine. Microwave assisted reaction were carried out in QPro-M microwave synthesizer. IR spectra were recorded Shimadzu FT-IR-8400 instrument using $\mathrm{KBr}$ pellet method. Mass spectra were recorded on Shimadzu GC-MS-QP2010 model using Direct Injection Probe technique. ${ }^{1} \mathrm{H}$ NMR was determined in DMSO- $d_{6}$ solution on a Bruker Ac $400 \mathrm{MHz}$ spectrometer. Elemental analysis of the all the synthesized compounds was carried out on Elemental Vario EL III Carlo Erba 1108 model and the results are in agreements with the structures assigned.

Synthesis of 4, 4, 4-trifluoro-1-(aryl) butane-1, 3-dione

Synthesis of 4, 4, 4-trifluoro-1-(aryl) butane-1, 3-dione was achieved using previously published methods ${ }^{21}$. 
General procedure for the synthesis of (5-(trifluoromethyl)-4, 7-dihydro-7-(aryl)-2(methylthio)-[1, 2, 4] triazolo [1, 5-a] pyrimidin-6-yl) (4-methoxyphenyl) methanone (PK- 101 to 110)

A mixture of the 5-(methylthio)-2H-1,2,4-triazol-3-amine (0.01 mol), 4,4,4-trifluoro-1-(4methoxyphenyl)butane-1,3-dione $(0.01 \mathrm{~mol})$ and an appropriate aromatic aldehyde $(0.01$ $\mathrm{mol})$ in ethanol $(5 \mathrm{~mL})$ was irradiated under microwave conditions at $120{ }^{\circ} \mathrm{C}$ for $10-15 \mathrm{~min}$. The microwave irradiation was operated in 30 second cycles. The reaction mixture was allowed to stand overnight at room temperature and was then filtered to give the solid triazolopyrazolopyrimidine products PK 101-110, which were washed with ethanol and dried in air. Triazolopyrimidines were obtained in high purity and did not require further purification by recrystallization.

(5-(Trifluoromethyl)-4,7-dihydro-7-(4-methoxyphenyl)-2-(methylthio)-[1,2,4] triazolo [1,5-a]pyrimidin-6-yl)(4-methoxyphenyl)methanone (PK-101)<smiles>COc1ccc(C(=O)C2=C(C(F)(F)F)Nc3nc(SC)nn3C2c2ccc(OC)cc2)cc1</smiles>

Figure 1. Structure of PK-101

Yield: 78\%; $\mathrm{mp} 228-230^{\circ} \mathrm{C}$; IR $\left(\mathrm{cm}^{-1}\right): 3265$ (N-H stretching of secondary amine), 3022 (C-H stretching of aromatic ring), 2920 (C-H asymmetrical stretching of $\mathrm{CH}_{3}$ group), 2866 (C-H asymmetrical stretching of $\mathrm{CH}_{3}$ group), 1660 ( $\mathrm{C}=\mathrm{O}$ stretching of carbonyl group), $1620(\mathrm{C}=\mathrm{N}$ stretching of triazole ring $), 1555(\mathrm{~N}-\mathrm{H}$ deformation of pyrimidine ring), 1513, 1481 and $1440(\mathrm{C}=\mathrm{C}$ stretching of aromatic ring), 1414 (C-H asymmetrical deformation of $\mathrm{CH}_{3}$ group), 1328 (C-H symmetrical deformation of $\mathrm{CH}_{3}$ group), 1282 (C-N stretching), 1245 (C-O-C stretching), 1031 (C-H in plane deformation of aromatic ring), 827 (C-H out of plane bending of 1,4-disubstituion);

${ }^{1} \mathrm{H}$ NMR (DMSO- $\left.d_{6}\right) \delta$ ppm: $2.47\left(3 \mathrm{H}, \mathrm{SCH}_{3}\right), 3.72\left(\mathrm{~s}, 3 \mathrm{H}, \mathrm{H}_{\mathrm{aa}}{ }^{\circ}\right), 6.56\left(\mathrm{~s}, 1 \mathrm{H}, \mathrm{H}_{\mathrm{b}}\right), 6.75-$ $6.78\left(\mathrm{~d}, 2 \mathrm{H}, \mathrm{H}_{\mathrm{cc}^{\prime}}, J=8.84 \mathrm{~Hz}\right), 6.96-7.00\left(\mathrm{t}, 2 \mathrm{H}, \mathrm{H}_{\mathrm{dd}^{\prime}}\right), 7.32-7.38\left(\mathrm{~m}, 4 \mathrm{H}, \mathrm{H}_{\mathrm{ee}} \cdot \mathrm{ff}^{\prime}\right), 7.57(\mathrm{~s}$, $1 \mathrm{H}, \mathrm{H}_{\mathrm{g}}$ ), $11.06\left(\mathrm{~s}, 1 \mathrm{H}, \mathrm{H}_{\mathrm{h}}\right)$; MS: $\mathrm{m} / z$ 476; Anal. Calcd. for $\mathrm{C}_{22} \mathrm{H}_{19} \mathrm{~F}_{3} \mathrm{~N}_{4} \mathrm{O}_{3} \mathrm{~S}: \mathrm{C}, 58.61 ; \mathrm{H}$, 3.98; N, 13.02. Found: C, 58.54; H, 3.88; N, $12.95 \%$.

(5-(Trifluoromethyl)-7-(4-fluorophenyl)-4,7-dihydro-2-(methylthio)-[1,2,4]triazolo [1,5-a]pyrimidin-6-yl)(4-methoxyphenyl)methanone (PK-102)<smiles>COc1ccc(C(=O)C2=C(C(F)(F)F)Nc3nc(SC)nn3C2c2ccc(F)cc2)cc1</smiles>

Figure 2. Structure of PK-102 
Yield: 72\%; mp 226-228 ${ }^{\circ} \mathrm{C}$; IR $\left(\mathrm{cm}^{-1}\right): 3232(\mathrm{~N}-\mathrm{H}$ stretching of secondary amine), 3116 (C-H symmetrical stretching of $\mathrm{CH}_{3}$ group), 2935 (C-H asymmetrical stretching of $\mathrm{CH}_{3}$ group), $1715(\mathrm{C}=\mathrm{O}$ stretching of carbonyl group), $1645(\mathrm{C}=\mathrm{N}$ stretching of triazole ring), 1522 and 1481 ( $\mathrm{C}=\mathrm{C}$ stretching of aromatic ring), 1437 ( $\mathrm{C}-\mathrm{H}$ asymmetrical deformation of $\mathrm{CH}_{3}$ group), 1408 (C-N-C stretching of pyrimidine ring), 1340 (C-H symmetrical deformation of $\mathrm{CH}_{3}$ group), 1276 (C-N stretching of pyrimidine ring), 1240 (C-O-C asymmetrical stretching of ether linkage), 1172 (C-H in plane deformation of aromatic ring), 1060 (C-O-C symmetrical stretching of ether linkage), 868 (C-H out of plane deformation of 1,4-disubstitution).

${ }^{1} \mathrm{H}$ NMR (DMSO-d $\left.d_{6}\right) \delta$ ppm: $2.48\left(3 \mathrm{H}, \mathrm{SCH}_{3}\right), 3.79\left(\mathrm{~s}, 3 \mathrm{H}, \mathrm{H}_{\mathrm{a}}\right), 6.39\left(\mathrm{~s}, 3 \mathrm{H}, \mathrm{H}_{\mathrm{b}}\right), 6.94-6.97$ $\left(\mathrm{d}, 2 \mathrm{H}, \mathrm{H}_{\mathrm{cc}}, J=8.4 \mathrm{~Hz}\right), 7.03-7.09\left(\mathrm{~m}, 4 \mathrm{H}, \mathrm{H}_{\mathrm{dd}^{\prime}-e^{\prime}}\right), 7.71-7.73\left(\mathrm{~m}, 3 \mathrm{H}, \mathrm{H}_{\mathrm{ff}-\mathrm{g}}\right), 11.27\left(\mathrm{~s}, 1 \mathrm{H}, \mathrm{H}_{\mathrm{h}}\right) ; \mathrm{MS}: \mathrm{m} / \mathrm{z}$ 464; Anal. Calcd. For $\mathrm{C}_{21} \mathrm{H}_{16} \mathrm{~F}_{4} \mathrm{~N}_{4} \mathrm{O}_{2} \mathrm{~S}$ : C, 57.42; H, 3.37; N, 13.39. Found: C, 57.30; H, $3.25 ; \mathrm{N}, 13.22 \%$.

(5-(Trifluoromethyl)-4,7-dihydro-2-(methylthio)-7-p-tolyl-[1,2,4]triazolo[1,5a]pyrimidin-6-yl)(4-methoxyphenyl)methanone (PK-103)<smiles>COc1ccc(C(=O)C2=C(C(F)(F)F)Nc3nc(SC)nn3C2c2ccc(C)cc2)cc1</smiles>

Figure 3. Structure of PK-103

Yield: 53\%; mp 198-200 ${ }^{\circ} \mathrm{C}$; IR $\left(\mathrm{cm}^{-1}\right): 3261$ (N-H stretching of secondary amine), 3035 (C-H stretching of aromatic ring), 2922 (C-H asymmetrical stretching of $\mathrm{CH}_{3}$ group), 2876 (C-H asymmetrical stretching of $\mathrm{CH}_{3}$ group), 1670 ( $\mathrm{C}=\mathrm{O}$ stretching of carbonyl group), $1606(\mathrm{C}=\mathrm{N}$ stretching of triazole ring), $1550(\mathrm{~N}-\mathrm{H}$ deformation of pyrimidine ring), 1516 and $1482\left(\mathrm{C}=\mathrm{C}\right.$ stretching of aromatic ring), $1440\left(\mathrm{C}-\mathrm{H}\right.$ asymmetrical deformation of $\mathrm{CH}_{3}$ group), 1412 (C-H symmetrical deformation of $\mathrm{CH}_{3}$ group), 1330 (C-N stretching), 1249 (C-O-C stretching), 1029 (C-H in plane deformation of aromatic ring), 822 (C-H out of plane bending of 1,4-disubstituion);

${ }^{1} \mathrm{H}$ NMR (DMSO- $\left.d_{6}\right) \delta$ ppm: $2.15\left(\mathrm{~s}, 3 \mathrm{H}, \mathrm{H}_{\mathrm{a}}\right), 2.45\left(3 \mathrm{H}, \mathrm{SCH}_{3}\right), 3.81\left(\mathrm{~s}, 3 \mathrm{H}, \mathrm{H}_{\mathrm{b}}\right), 6.28(\mathrm{~s}$, $\left.1 \mathrm{H}, \mathrm{H}_{\mathrm{c}}\right), 6.85-6.87\left(\mathrm{~d}, 2 \mathrm{H}, \mathrm{H}_{\mathrm{dd}}, J=8.80 \mathrm{~Hz}\right), 6.94-6.97\left(\mathrm{~d}, 2 \mathrm{H}, \mathrm{H}_{\mathrm{ee}}, J=9.20 \mathrm{~Hz}\right), 7.03-7.05(\mathrm{~d}$, $\left.2 \mathrm{H}, \mathrm{H}_{\mathrm{ff}}, J=8.00 \mathrm{~Hz}\right), 7.71-7.74\left(\mathrm{~d}, 3 \mathrm{H}, \mathrm{H}_{\mathrm{gg}}{ }^{\prime} \mathrm{h}\right), 11.26\left(\mathrm{~s}, 1 \mathrm{H}, \mathrm{H}_{\mathrm{i}}\right)$; MS: $m / z$ 460; Anal. Calcd. For $\mathrm{C}_{22} \mathrm{H}_{19} \mathrm{~F}_{3} \mathrm{~N}_{4} \mathrm{O}_{2} \mathrm{~S}$ : C, 60.87; H, 4.14; N, 13.52. Found: C, 60.79; H, 4.02; N, $13.40 \%$.

(7-(4-Chlorophenyl)-5-(trifluoromethyl)-4,7-dihydro-2-(methylthio)-[1,2,4]triazolo [1,5-a]pyrimidin-6-yl)(4-methoxyphenyl)methanone (PK-105)<smiles>COc1ccc(C(=O)C2=C(C(F)(F)F)Nc3nc(SC)nn3C2c2ccc(Cl)cc2)cc1</smiles>

Figure 4. Structure of PK-105 
Yield: 75\%; mp 240-242 ${ }^{\circ} \mathrm{C}$; IR $\left(\mathrm{cm}^{-1}\right)$ : 3219 (N-H stretching of secondary amine), 3048 (C-H stretching of aromatic ring), 2965 (C-H asymmetrical stretching of $\mathrm{CH}_{3}$ group), 2874 (C-H asymmetrical stretching of $\mathrm{CH}_{3}$ group), $1666(\mathrm{C}=\mathrm{O}$ stretching of carbonyl group), $1595(\mathrm{C}=\mathrm{N}$ stretching of triazole ring), 1516 ( N-H deformation of pyrimidine ring), $1440,1400\left(\mathrm{C}=\mathrm{C}\right.$ stretching of aromatic ring), $1411\left(\mathrm{C}-\mathrm{H}\right.$ asymmetrical deformation of $\mathrm{CH}_{3}$ group), 1344 (C-H symmetrical deformation of $\mathrm{CH}_{3}$ group), 1280 (C-N stretching), 1248 (CO-C stretching), 1033 (C-H in plane deformation of aromatic ring), 815 (C-H out of plane bending of 1,4-disubstituion);

${ }^{1} \mathrm{H}$ NMR (DMSO- $\left.d_{6}\right) \delta$ ppm: 2.48(3H, $\left.\mathrm{SCH}_{3}\right), 3.81\left(\mathrm{~s}, 3 \mathrm{H}, \mathrm{H}_{\mathrm{a}}\right), 6.39\left(\mathrm{~s}, 3 \mathrm{H}, \mathrm{H}_{\mathrm{b}}\right), 6.95-$ $6.97\left(\mathrm{~d}, 2 \mathrm{H}, \mathrm{H}_{\mathrm{cc}}, J=8.40 \mathrm{~Hz}\right), 7.07-7.09\left(\mathrm{~d}, 2 \mathrm{H}, \mathrm{H}_{\mathrm{dd}}, J=8.00 \mathrm{~Hz}\right), 7.30-7.32\left(\mathrm{~d}, 2 \mathrm{H}, \mathrm{H}_{\mathrm{ee}}, J\right.$ $=8.00 \mathrm{~Hz}), 7.74-7.80\left(\mathrm{~m}, 3 \mathrm{H}, \mathrm{H}_{\mathrm{ff}}-\mathrm{g}\right), 11.36\left(\mathrm{~s}, 1 \mathrm{H}, \mathrm{H}_{\mathrm{h}}\right) ; \mathrm{MS}: \mathrm{m} / \mathrm{z}$ 480; Anal. Calcd. for $\mathrm{C}_{21} \mathrm{H}_{16} \mathrm{ClF}_{3} \mathrm{~N}_{4} \mathrm{O}_{2} \mathrm{~S}: \mathrm{C}, 55.25 ; \mathrm{H}, 3.25 ; \mathrm{N}, 12.89$. Found: C, 55.10; H, 3.12; N, $12.76 \%$.

\section{IR spectral study}

IR spectra were recorded on Shimadzu FT-IR-8400 model using KBr pellet method. Various functional groups present in molecule were identified by characteristic frequency obtained for them. For triazolopyrimidines PK-101 to 110 , confirmatory bands for secondary amine and carbonyl groups were observed at $3159-3279 \mathrm{~cm}^{-1}$ and $1658-1712 \mathrm{~cm}^{-1}$ respectively. Another characteristic $\mathrm{C}=\mathrm{N}$ stretching band of triazole ring was observed at $1521-1641 \mathrm{~cm}^{-1}$, which suggested formation of desired products VP-101 to 110 .

\section{${ }^{1}$ H NMR spectral study}

${ }^{1} \mathrm{H}$ NMR spectra were recorded in DMSO- $d_{6}$ solution on a Bruker Ac $400 \mathrm{MHz}$ spectrometer using TMS as an internal standard. Number of protons and their chemical shifts were found to support the structure of the synthesized compounds.

${ }^{1} \mathrm{H}$ NMR spectra confirmed the structures of triazolopyrimidines PK-101 to 110 on the basis of following signals: a singlet for the methine proton of pyrimidine ring at 6.28-6.72 $\delta$ ppm, a singlet for the methine proton of triazole ring at 7.27-7.87 $\delta \mathrm{ppm}$ and singlet for secondary amine group of pyrimidine proton at 11.05-11.46 $\delta \mathrm{ppm}$, respectively.

\section{Biological evaluation}

Antimicrobial evaluation

All of the synthesized compounds (PK-101 to 110) were tested for their antibacterial and antifungal activity (MIC) in vitro by broth dilution method with two Gram-positive bacteria Staphylococcus aureus MTCC-96, Streptococcus pyogenes MTCC 443, two Gram-negative bacteria Escherichia coli MTCC 442, Pseudomonas aeruginosa MTCC 441 and three fungal strains Candida albicans MTCC 227, Aspergillus Niger MTCC 282, Aspergillus clavatus MTCC 1323 taking gentamycin, ampicillin, chloramphenicol, ciprofloxacin, norfloxacin, nystatin and greseofulvin as standard drugs. The standard strains were procured from the Microbial Type Culture Collection (MTCC), Institute of Microbial Technology, Chandigarh, India.

The minimal inhibitory concentration (MIC) values (Table 1) for all the newly synthesized compounds, defined as the lowest concentration of the compound preventing the visible growth, were determined by using micro dilution broth method according to NCCLS standards $^{22}$. 
Table 1. Antibacterial and antifungal activity of synthesized compounds PK-101 to 110

\begin{tabular}{cccccccc}
\hline & \multicolumn{7}{c}{ Minimum inhibition concentration, $\mu \mathrm{g} \mathrm{mL} \mathrm{mL}^{-1}$} \\
\hline \multirow{2}{*}{ Code } & Gram-positive & \multicolumn{7}{l}{ Gram-negative } & Fungal species & \\
\cline { 2 - 8 } & S.a. & S. $p$. & E.c. & $P . a$. & $C . a$. & $A . n$. & A.c. \\
\hline PK-101 & 500 & 500 & 1000 & $>1000$ & 1000 & 500 & 125 \\
PK-102 & 125 & 100 & 50 & 250 & 500 & 1000 & 500 \\
PK-103 & 100 & 1000 & 250 & 1000 & 1000 & 500 & 1000 \\
PK-104 & 50 & 500 & 250 & 250 & $>1000$ & 1000 & $>1000$ \\
PK-105 & 500 & 1000 & 500 & 1000 & 500 & 500 & 100 \\
PK-106 & 125 & 25 & 100 & 100 & 500 & $>1000$ & 500 \\
PK-107 & 250 & 1000 & 1000 & 250 & 500 & 1000 & 1000 \\
PK-108 & 100 & 250 & 100 & 500 & 500 & 1000 & 1000 \\
PK-109 & 250 & 125 & 250 & 250 & 100 & 1000 & 250 \\
PK-110 & 62.5 & 100 & 100 & 250 & 250 & 500 & 250 \\
Ampicillin & 250 & 100 & 100 & 100 & - & - & - \\
Chloramphenicol & 50 & 50 & 50 & 50 & - & - & - \\
Ciprofloxacin & 50 & 50 & 25 & 25 & - & - & - \\
Norfloxacin & 10 & 10 & 10 & 10 & - & - & - \\
Nystatin & - & - & - & - & 100 & 100 & 100 \\
Griseofulvin & - & - & - & - & 500 & 100 & 100 \\
\hline
\end{tabular}

Acknowledgement

The authors are thankful to the Shri R.R. Lalan College for providing necessary facilities.

\section{References}

1. Leadbeater N, Chemistry World, 2004, 1, 38-41.

2. Fischer G, Adv Heterocycl Chem., 1993, 57, 81-138; DOI:10.1016/S00652725(08)60887-9

3. Shaban M A E and Morgan A E A, Adv Heterocycl Chem., 2000, 77, 345-394; DOI:10.1016/S0065-2725(00)77009-7

4. Shaban M A E and Morgan A E A, Adv Heterocycl Chem., 2000, 73, 131-176; DOI:10.1016/S0065-2725(08)60942-3

5. Shaban M A E and Morgan A E A, Adv Heterocycl Chem., 2000, 75, 243-281; DOI:10.1016/S0065-2725(08)60986-1

6. Zhang N, Semiramis A K, Thai N, Jay A, Richard H, Judy L, James G and Carl B, J Med Chem., 2007, 50(2), 319-327; DOI:10.1021/jm060717i

7. Havlicek L, Fuksova K, Krystof V, Orsag M, Vojtesek B and Strnad M, Bioorg Med Chem., 2005, 13(18), 5399-5407; DOI:10.1016/j.bmc.2005.06.007

8. Fraley M E, Hoffman W F and Rubino R S, Bioorg Med Chem Lett., 2002, 12(19), 2767-2770; DOI:10.1016/S0960-894X(02)00525-5

9. Chen Q, Zhu X L, Liu Z M and Guang-Fu Yang, Eur J Med Chem., 2008, 43(3), 595603; DOI:10.1016/j.ejmech.2007.04.021

10. Uryu S, Tokuhiro S, Murasugi T and Tomiichiro Oda, Brain Res., 2002, 946(2), 298306; DOI:10.1016/S0006-8993(02)02898-6

11. Liekfeld H, Pharmazeut Ztg., 1994, 139(1), 34.

12. Yamashkin S A, Kucherenko N Y, Yurovskaya M A, Chem Heterocycl Compd (Engl Transl.), 1997, 33(5), 499-514; DOI:10.1007/BF02291929 
13. Krasovsky A L, Moiseev A M, Nenajdenko V G and Balenkova E S, Synthesis, 2002, 901-911.

14. Hammouda M H, Etman E M and Metwally A, J Serb Chem Soc., 1992, 57, 165.

15. Al-Schiekh M A, El-Din, A M S, Hafez E A and Elnagdi M H, J Chem Res., 2004, 174.

16. Kuznetsova O A, Filyakova V I, Pashkevich K I, Ulomskii E N, Plekhanov P V, Rusinov G L, Kodess M I and Rusinov V L, Russ Chem Bull (Engl Transl.,) 2003, 52, 1190-1194; DOI:10.1023/A:1024777828344

17. Lipunova G N, Nosova E V, Kodess M I, Charushin V N, Rozin Y A and Chasovskikh O M, Russ J Org Chem (Engl Transl.), 2001, 37(4), 570-576; DOI:10.1023/A:1012498322793

18. Hassaneen H M, Abdallah T A, Abdelhadi H A, Hassaneen H M E and Pagni R M, Heteroat Chem., 2003, 14(6), 491-497; DOI:10.1002/hc.10166

19. Al-Zaydi K M, Borik R M and Elnagdi M H, Molecules, 2003, 8(12), 910-923; DOI:10.3390/81200910

20. Kanno H, Yamaguchi H, Ichikawa Y and Isoda S, Chem Pharm Bull., 1991, 39(5), 1099-1105; DOI:10.1248/cpb.39.1099

21. Jeon S L, Kim D H, Son J B and Jeong I H, Bulletin Korean Chem Soc., 2006, 27(12), 1961-1962; DOI:10.5012/bkcs.2006.27.12.1961

22. Zgoda J R and Porter J R, Pharm Biol., 2001, 39(3), 221-225; DOI:10.1076/phbi.39.3.221.5934 